\title{
Efeito do azul de metileno na resposta inflamatória e hemodinâmica em pacientes submetidos à cirurgia de revascularização miocárdica com circulação extracorpórea
}

\author{
Methylene blue use in coronary artery bypass surgery: a prospective randomized study of the \\ hemodynamic and inflammatory responses
}

Nilzo Augusto Mendes RIBEIRO, Noedir Antônio Groppo STOLF, Augusto Ferreira da SILVA JUNIOR, Valcellos José da Cruz VIANA, Eduardo Novaes de CARVALHO, Rodrigo ATHANÁZIO, Mitermayer Galvão REIS, Sérgio Almeida de OLIVEIRA

RBCCV 44205-664

Resumo

Objetivo: Estudar em pacientes submetidos à cirurgia de revascularização miocárdica com circulação extracorpórea os efeitos da infusão de azul de metileno na reação inflamatória sistêmica e nas condições hemodinâmica e laboratorial.

Método: Foram estudados 60 pacientes randomizados em dois grupos, utilizando-se a infusão de azul de metileno em um dos dois grupos. Amostras de sangue foram colhidas antes da indução anestésica, 3, 6, 24 e 48 horas após o término da circulação extracorpórea para dosagens dos marcadores de inflamação (IL-1 $\beta$, IL-6, IL-8, IL-10, IL-12p40 e TNF $\alpha$ ), NO, gasometria e outras dosagens de rotina.
Resultados: O grupo que utilizou azul de metileno mostrou, em diferentes momentos das coletas, maior resistência vascular sistêmica, menor concentração de TNF $\alpha$, menor número de leucócitos e neutrófilos e menor nível de óxido nítrico. Não ocorreram efeitos adversos importantes.

Conclusões: A infusão de azul de metileno não evidenciou alterações clínicas ou pulmonares adversas, mostrando uma tendência menor à resposta inflamatória sistêmica, menores níveis de óxido nítrico e melhor estabilidade hemodinâmica.

Descritores: Revascularização miocárdica. Citocinas. Óxido nítrico. Azul de metileno.

Hospital Santa Izabel da Santa Casa de Misericórdia da Bahia

Instituto do Coração da FMUS

Endereço para correspondência: Nilzo A. M. Ribeiro. Hospital Santa Izabel. Praça Almeida Couto 500, Nazaré. Salvador, BA. CEP 40.050410. Tel/Fax (71) 326-4455

E-mail: nilzoribeiro@terra.com.br 


\section{Abstract}

Objective: To assess the effects of methylene blue infusion on on-pump coronary artery bypass patients in relation to hemodynamic, laboratorial and systemic inflammatory response.

Method: Sixty patients were randomized in two groups. Methylene blue was infused in one group. Blood samples were collected before the anesthesia and, 3, 6, 24 and 48 hours after the end of the extracoporeal circulation to determine the IL1 $\beta$, IL-6, IL-8, IL-10, IL-12p40, TNF $\alpha$ and nitric oxide levels and perform gasometry and other routine tests.

\section{INTRODUÇÃO}

A utilização de circulação extracórporea (CEC) em cirurgia cardíaca induz alterações inflamatórias conhecidas desde o início dos anos 70, conforme relato de PARKER et al. [1].

Diminuição na resistência vascular sistêmica associada à hipotensão arterial foi denominada por GOMES et al. [2] como síndrome vasoplégica, mantendo correlação com a SIRS.

Trabalhos recentes têm mostrado que a infusão de azul de metileno melhora as condições da resistência vascular sistêmica e pressão arterial, diminuindo ou até abolindo as necessidades do uso de catecolaminas [3-6].

SALARIS et al. [7] relatam a eficácia do azul de metileno na prevenção do dano dos radicais livres aos tecidos do fígado e rins num modelo in vitro de isquemia / reoxigenação.

Existindo, conforme FINKEL et al. [8], uma correlação moduladora entre citocinas e óxido nítrico e sendo o azul de metileno um bloqueador da produção de óxido nítrico, de acordo MAYER et al. [9], levanta-se a possibilidade de utilização dessa substância para prevenir a SIRS em cirurgia com CEC.

\section{OBJETIVO}

Estudar em pacientes submetidos à cirurgia de revascularização miocárdica com CEC, separados em dois grupos, um que recebeu azul de metileno (AM) e outro que não: A resposta inflamatória sistêmica através a determinação de interleucinas e óxido nítrico;

As condições hemodinâmicas e laboratoriais.

\section{MÉTODO}

Todos os pacientes assinaram termo de consentimento previamente aprovado pelo Conselho de Ética do Hospital Santa Izabel da Santa Casa de Misericórdia da Bahia e Faculdade de Medicina da Universidade de São Paulo.

A indução anestésica foi feita com midazolan, sufentanil e pancurânio e manutenção com sevoflurano.
Results: In the methylene group we observed at different stages, higher systemic vascular resistance, lower TNF $\alpha$ concentrations, fewer leukocytes and neutrophils as well as lower level of nitric oxide. No adverse effects were evidenced.

Conclusions: Methylene blue infusion showed less tendency of systemic inflammatory responses, lower levels of nitric oxide and a better hemodynamic performance.

Descriptors: Myocardial revascularization. Cytokines. Nitric oxide. Methylene blue.

Antibioticoterapia com cefalotina e metilprednisolona na dose de $30 \mathrm{mg} / \mathrm{kg}$ foram utilizadas.

Após medida dos parâmetros hemodinâmicos, iniciava-se a infusão de azul de metileno conforme randomização, na dosagem de $2 \mathrm{mg} / \mathrm{kg}$ de peso diluído em solução de glicose a $5 \%$ durante 6 horas (ampolas de $5 \mathrm{ml} \mathrm{a} \mathrm{1 \%} \mathrm{-} \mathrm{Farmácia} \mathrm{do} \mathrm{Hospital}$ das Clínicas da Faculdade de Medicina da Universidade de São Paulo), independentemente do tempo cirúrgico.

A operação foi realizada com circulação CEC e hipotermia leve/moderada com utilização de cardioplegia normotérmica anterógrada.

As coletas de sangue foram realizadas antes da indução, $3,6,24$ e 48 horas após o término da CEC. As amostras para as dosagens das citocinas IL- $1 \beta$, IL-6, IL- 8 , IL-10 e TNF $\alpha$ foram encaminhadas ao Laboratório de Patologia e Biologia Molecular da Fundação Osvaldo Cruz/Bahia e para a dosagem de NO ao Laboratório de Inflamação e Dor da Faculdade de Medicina de Ribeirão Preto.

Outras dosagens de rotina foram realizadas no Hospital Santa Izabel.

\section{Análise Estatística}

Os dados foram armazenados e analisados no SPSS 10.0. As variáveis contínuas foram apresentadas sob forma de medidas de tendência (média aritmética) e medidas de dispersão (desvios padrão -DP). Utilizou-se o teste $\mathrm{T}$ de Student para comparação de médias e o teste não paramétrico de Mann-Whitney para comparação das médias quando as variâncias não eram homogêneas. Para a comparação estatística das proporções utilizou-se o teste Qui-quadrado $\left(\chi^{2}\right)$ ou o teste exato de Fisher, esse último quando as amostras estratificadas tornavam-se muito pequenas. Estabeleceu-se um nível de significância de 5\%.

\section{RESULTADOS}

\section{Caracterização dos pacientes e dados intra-} operatórios.

Todos os pacientes foram avaliados quanto idade, sexo, 
revascularização miocárdica com circulação extracorpórea

raça, tabagismo, hipertensão arterial, diabetes, angina estável, angina instável, infarto prévio, tempo de CEC, tempo de pinçamento da aorta e número de pontes realizadas.

Não ocorreram diferenças significativas nessas variáveis.

\section{Queixas pós-operatórias}

As variáveis avaliadas foram: urina e/ou fezes azuladas, diarréia, náuseas, cefaléia, tontura, astenia e dispnéia. Urina azulada permanecendo por até quatro dias de pós-operatório foi encontrada em $37,9 \%$ dos pacientes do grupo que usou AM $(p<0,05)$ e em nenhum do grupo controle foi a única com diferença com significância estatística. Todas as outras variáveis não mostraram diferenças significativas.

\section{Variáveis laboratoriais}

Valores maiores com significância estatística foram encontrados na contagem de leucócitos e neutrófilos, nos grupos controle e experimental (AM), na $48^{\text {a }}$ hora. Estes valores foram, respectivamente, $16.311 \pm 3.974$ contra 13.180 \pm 4.355 e $13.782 \pm 3.854$ contra $10.883 \pm 3.723$.

Em todas as outras variáveis não foram encontradas diferenças significativas (Figuras 1 e 2).

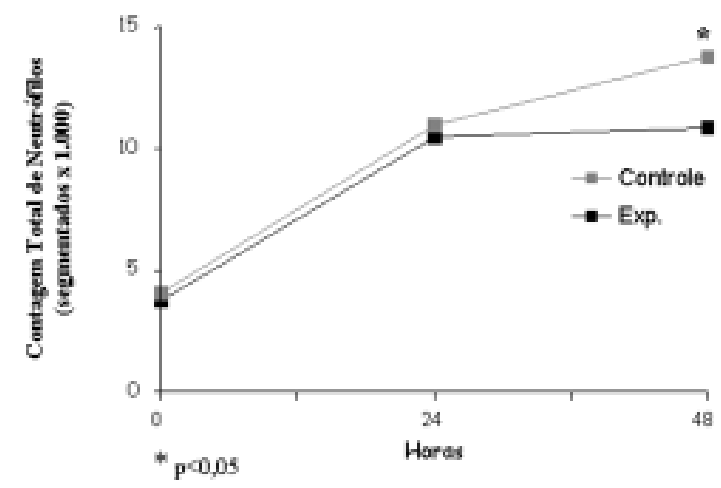

Fig. 1 - Contagem de neutrófilos

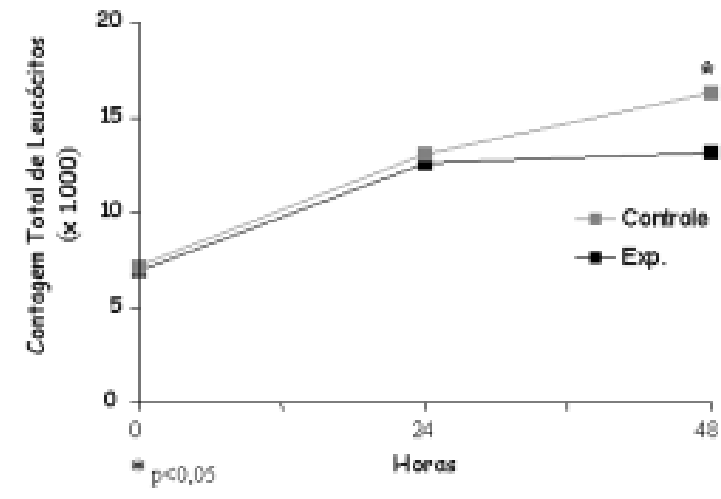

Fig. 2 - Contagem de leucócitos

\section{Variáveis gasométricas, $\mathrm{pH}, \mathrm{Na}^{+} \mathrm{e} \mathrm{K}^{+}$}

As variáveis determinadas ou calculadas não mostraram diferenças nos dois grupos, exceto em relação ao $\mathrm{K}+$ na $48^{\mathrm{a}}$ hora, porém ainda na faixa da normalidade.

\section{Variáveis hemodinâmicas}

A pressão arterial sistêmica diastólica (PASD) foi maior no grupo com AM do que no grupo controle $(67,0 \mathrm{mmHg} \pm$ $12,6$ contra $59,5 \mathrm{mmHg} \pm 12,2 \mathrm{p}<0,05)$ na amostra da $3^{\mathrm{a}}$ hora. A resistência vascular sistêmica (RVS) foi maior no grupo AM do que no grupo controle na amostra da $6^{\text {a }}$ hora $(987,4$ dyn.s.cm $\mathrm{cm}^{-5} \pm 414,8$ contra 764,4 dyn.s.cm ${ }^{-5} \pm 349,1 \mathrm{p}<0,05$ ). Não ocorreram diferenças significantes nas outras variáveis (Figuras 3 e 4)

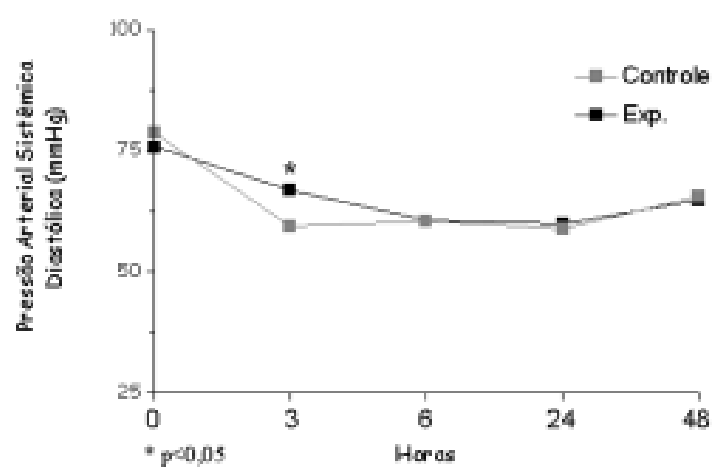

Fig. 3 - Pressão arterial sistêmica diastólica

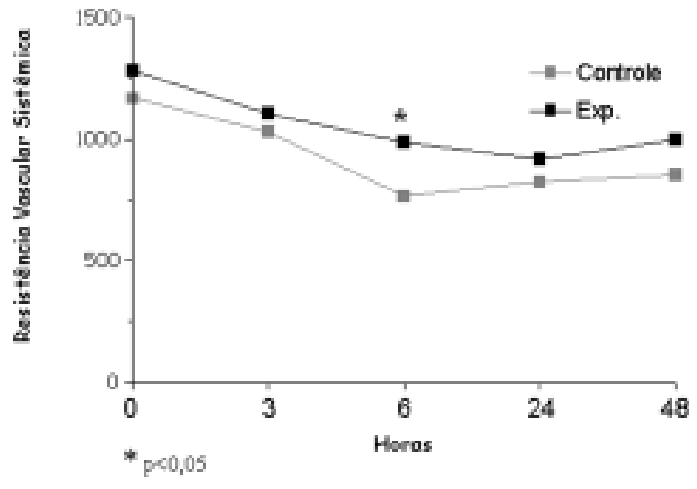

Fig. 4 - Resistência vascular sistêmica

\section{Variáveis imunomoleculares e óxido nítrico}

As dosagens de IL-1- $\beta$, IL-6, IL-8, IL-10, e IL-12p40 não mostraram diferenças significativas entre os dois grupos de pacientes estudados (Figuras 5, 6, 7 e Tabela 1). 


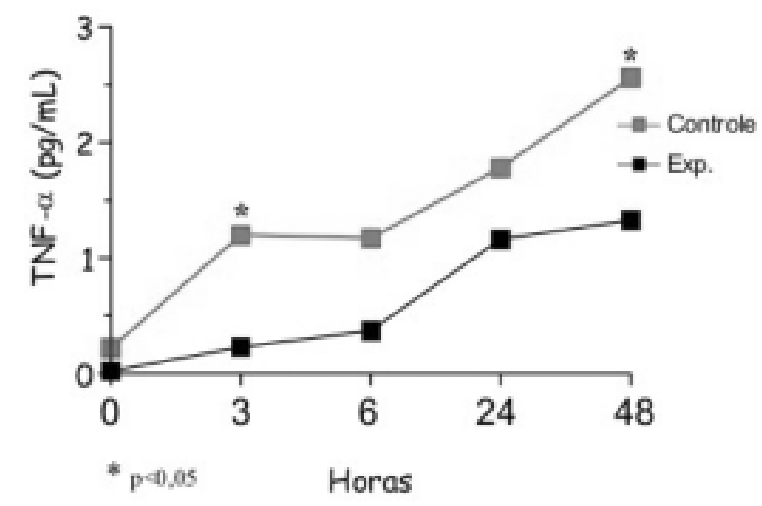

Fig. 5 - Valores de TNF $\alpha$

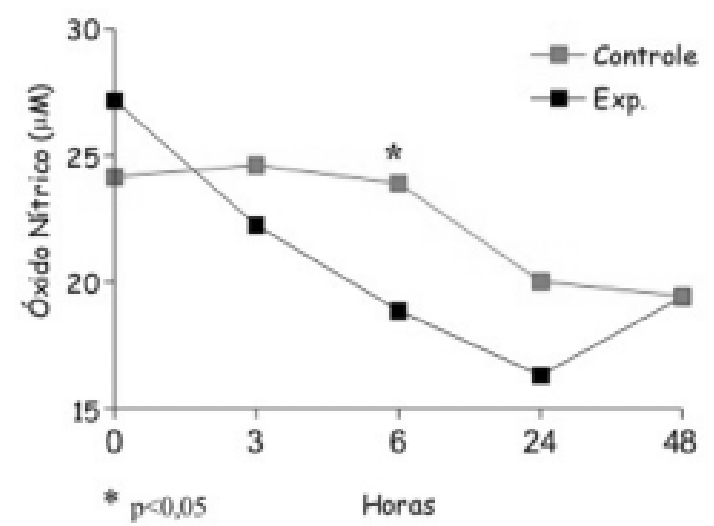

Fig. 6 - Nível de óxido nítrico

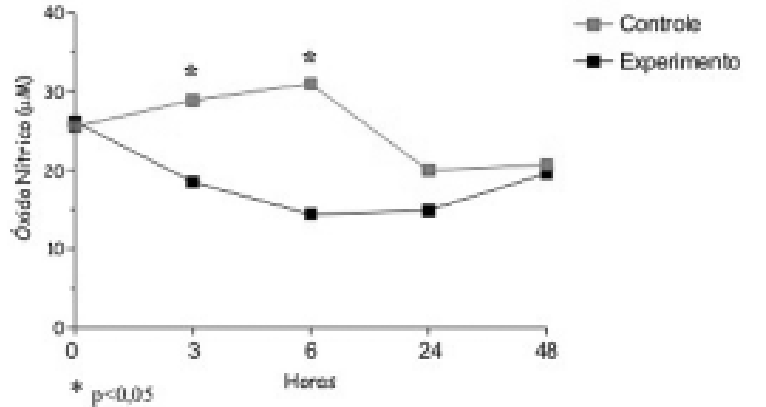

Fig. 7 - Nível de óxido nítrico em pacientes que não fizeram uso de vasodilatadores no intra-operatório

O TNF $\alpha$ mostrou diferença significativa na $3^{\mathrm{a}}$ e $48^{\mathrm{a}}$ hora pós-CEC, sendo maior no grupo controle (Figura 5 e Tabela 1).

O óxido nítrico mostrou diferença significativa na $6^{\mathrm{a}}$ hora após o término de CEC, sendo maior no grupo controle. Naqueles pacientes que não fizeram uso de drogas vasodilatadoras no período operatório, o óxido nítrico foi maior no grupo controle na $3^{\mathrm{a}}$ e $6^{\mathrm{a}}$ hora pós-CEC com significância estatística $(\mathrm{p}<0,05)$ Figuras 6 e 7 e Tabela 1.

\section{COMENTÁRIOS}

Os efeitos deletérios da CEC, com alterações das funções cerebrais, pulmonares, cardíacas, renais, hepáticas, coagulação e da resistência vascular sistêmica, são bem conhecidos e têm etiopatogenia variada [1,10-17].

Tabela 1. Variações da citocinas (pg/ml) E NO $(\mu \mathrm{m})$

\begin{tabular}{|c|c|c|c|c|c|c|c|}
\hline & TNF- $\alpha$ & IL-1 & IL-6 & IL-8 & IL-10 & IL-12 & $\mathrm{NO}$ \\
\hline \multicolumn{8}{|l|}{ Indução } \\
\hline Controle & $0,22 \pm 0,09$ & $0,85 \pm 0,37$ & $3,35 \pm 0,52$ & $10,50 \pm 1,05$ & $81,5 \pm 45,7$ & $24,71 \pm 4,39$ & $23,92 \pm 2,54$ \\
\hline $\mathrm{AM}$ & $0,02 \pm 0,01$ & $0,53 \pm 0,29$ & $2,90 \pm 0,43$ & $8,81 \pm 1,21$ & $119,4 \pm 24,4$ & $25,31 \pm 4,60$ & $26,96 \pm 3,53$ \\
\hline \multicolumn{8}{|l|}{3 horas } \\
\hline Controle & $1,20 \pm 0,41^{*}$ & $8,56 \pm 4,57$ & $74,4 \pm 9,85$ & $47,73 \pm 9,81$ & $1.082,0 \pm 143,0$ & $44,48 \pm 8,93$ & $25,03 \pm 2,81$ \\
\hline $\mathrm{AM}$ & $0,23 \pm 0,10^{*}$ & $8,05 \pm 3,56$ & $88,78 \pm 16,08$ & $39,72 \pm 6,00$ & $1.221,0 \pm 345,3$ & $65,34 \pm 24,41$ & $21,11 \pm 2,72$ \\
\hline \multicolumn{8}{|l|}{6 horas } \\
\hline Controle & $1,17 \pm 0,38$ & $4,03 \pm 2,10$ & $66,16 \pm 8,39$ & $52,84 \pm 16,00$ & $726,0 \pm 143,0$ & $30,42 \pm 4,76$ & $23,29 \pm 3,53 *$ \\
\hline $\mathrm{AM}$ & $0,37 \pm 0,23$ & $3,41 \pm 1,17$ & $57,05 \pm 7,59$ & $40,04 \pm 6,04$ & $852,5 \pm 196,8$ & $37,54 \pm 7,90$ & $18,49 \pm 2,75^{*}$ \\
\hline \multicolumn{8}{|l|}{24 horas } \\
\hline Controle & $1,78 \pm 0,44$ & $11,23 \pm 5,64$ & $42,74 \pm 5,79$ & $26,87 \pm 5,25$ & $128,2 \pm 24,4$ & $25,62 \pm 4,58$ & $19,05 \pm 2,31$ \\
\hline $\mathrm{AM}$ & $1,16 \pm 0,40$ & $9,20 \pm 4,09$ & $45,02 \pm 6,19$ & $36,05 \pm 4,45$ & $922,3 \pm 620,9$ & $25,18 \pm 5,02$ & $16,42 \pm 1,92$ \\
\hline \multicolumn{8}{|l|}{48 horas } \\
\hline Controle & $2,56 \pm 0,58^{*}$ & $7,83 \pm 3,37$ & $29,26 \pm 3,98$ & $31,65 \pm 8,45$ & $483,2 \pm 351,3$ & $23,96 \pm 3,22$ & $17,91 \pm 2,11$ \\
\hline AM & $1,33 \pm 0,48 *$ & $6,01 \pm 2,62$ & $29,32 \pm 3,45$ & $28,15 \pm 6,68$ & $645,3 \pm 527,9$ & $26,82 \pm 3,82$ & $19,74 \pm 2,28$ \\
\hline
\end{tabular}

$*_{\mathrm{p}}<0,05$ 
A síndrome da reação inflamatória sistêmica é hoje considerada um dos fatores mais importantes na gênese dessas alterações [10,14-16,18].

$\mathrm{O}$ azul de metileno tem sido relatado na literatura como sendo uma droga capaz de reverter a hipotensão arterial importante não responsiva às catecolaminas, diminuindo e até abolindo sua necessidade. Foi inicialmente utilizado no tratamento da hipotensão arterial do choque séptico e, mais recentemente, em pacientes submetidos à cirurgia cardíaca com quadro clínico de vasoplegia [3-6,19-21].

$\mathrm{O}$ óxido nítrico mostra-se em níveis elevados, tanto no choque séptico como na vasoplegia pós-cirúrgica [20-23], embora BRETT et al. [24] não relatem aumento pós-cirurgia cardíaca. O aumento dos níveis de óxido nítrico e das citocinas se relaciona à disfunção dos vários sistemas [11-13].

O azul de metileno, por meio de vários mecanismos como a inibição da ação do óxido nítrico na musculatura lisa vascular e diminuição da lesão de isquemia/reperfusão, poderia ter efeitos benéficos nos pacientes submetidos à cirurgia cardíaca com CEC [3-7,9,20].

A resposta inflamatória sistêmica desencadeada pela via clássica e alternativa tem como um dos fatores desencadeantes a isquemia/reperfusão, levando à ação no endotélio que regula o tônus vascular através a produção de óxido nítrico, prostaciclina, tromboxane e endotelina. $\mathrm{O}$ azul de metileno poderia, portanto, prevenir a SIRS ou diminuir os seus efeitos vasculares.

O presente estudo prospectivo e randomizado foi programado para avaliar os efeitos do azul de metileno em pacientes submetidos a cirurgia de revascularização miocárdica com CEC. Os dados pré-operatórios, demográficos e intra-operatórios foram comparados e não apresentaram diferenças estatisticamente significativas, demonstrando a eficiência da randomização.

Quanto a efeitos adversos do AM não houve diferenças em alterações clínicas entre os grupos, exceto urina azulada. Do mesmo modo, na avaliação laboratorial observou-se apenas nível mais elevado de $\mathrm{K}+$ no período de 24 horas no grupo AM porém ainda dentro da faixa da normalidade. Quanto a outras alterações especialmente pulmonares que poderiam ocorrer, não houve diferenças entre os valores das pressões pulmonares e resistência vascular pulmonar e gasometria Verifica-se, portanto, que não ocorreram efeitos adversos significativos. Na literatura, os efeitos do AM na função e na vasculatura pulmonares são controversos. ANDRADE et al. [3] observaram, entre seis pacientes tratados com AM, que a RVP aumentou em três, permaneceu inalterada em dois e diminuiu em um. KOELZOW et al. [5] estudaram dois grupos de pacientes transplantados hepáticos com e sem o uso de AM e verificaram que as pressões e resistência pulmonares aumentaram em ambos os grupos após o procedimento, mas não houve diferença entre os grupos em relação a esses parâmetros. PREISER et al. [21], em pacientes em choque séptico que receberam AM, relatam que não houve alteração da pressão pulmonar bem como dos gases arteriais, oferta e consumo de oxigênio. No entanto, WEINGARTNER et al. [23] verificaram leve aumento do índice de resistência vascular pulmonar e piora da função pulmonar, sem contudo ocorrerem alterações do enchimento e débito cardíaco.

$\mathrm{Na}$ avaliação da SIRS, no presente estudo, verificou-se que tanto as citocinas pró-inflamatórias (IL-1 $\beta$, IL-6, IL-8 e $\mathrm{TNF} \alpha$ ) bem como as antiinflamatórias (IL-10 e IL-12p40) mostraram aumento nos dois grupos, demonstrando a presença de SIRS após cirurgia cardíaca com CEC. Esses achados estão de acordo com a literatura $[13,25]$.

Quanto ao estudo comparativo das citocinas nos dois grupos, que é um dos objetivos do estudo, observou-se que não houve diferença significativa entre os grupos em nenhum período em relação à IL1 $\beta$, IL-6, IL-8, IL-10 e IL$12 \mathrm{p} 40$. As dosagens do TNF $\alpha$ mostraram valores mais baixos no grupo AM nos períodos de três a 48 horas, com significância estatística nos períodos de três e 48 horas, mostrando que o AM diminui a produção desta mas não das outras citocinas. Não se encontraram referências na literatura quanto à influência do AM na SIRS.

$\mathrm{Na}$ avaliação do leucograma verificou-se, no presente estudo, que ocorreu número absoluto significativamente menor dos leucócitos e neutrófilos no grupo AM ao fim de 48 horas. Embora isoladamente, esse achado não seja definitivo, ele sugere uma menor SIRS no grupo AM.

Em relação à avaliação hemodinâmica, na presente investigação, verificou-se que a resistência vascular sistêmica esteve maior em todos os períodos no grupo AM alcançando significância estatística na $6^{\mathrm{a}}$ hora. No mesmo sentido, a pressão arterial sistêmica diastólica foi significativamente maior na $3^{\text {a }}$ hora no grupo AM. Esses achados mostram melhor tônus vascular arterial como é esperado pelo mecanismo de ação do AM e a experiência acumulada da literatura em várias condições clínicas como o choque séptico e inclusive cirurgia cardíaca [3-6,21,23].

A determinação do óxido nítrico, na presente investigação, mostrou valores mais baixos no grupo AM de três a 24 horas após a CEC, alcançando significância estatística na $6^{a}$ hora. Quando se avaliou o grupo de pacientes que não recebeu vasodilatadores, a diferença foi significativa nos períodos de três a seis horas. Esses achados são esperados uma vez que o efeito do AM é conhecido, diminuindo ou inibindo a síntese de óxido nítrico através do discutível mecanismo inibição da óxido nítrico sintase. O efeito comprovado do AM é a inibição da guanilil ciclase, impedindo o aumento do cGMP ou atuando como receptor artificial de elétrons, desta maneira inibindo a formação de radicais livres [7,9]. Os níveis menores de óxido 
nítrico poderiam explicar os valores maiores da resistência vascular sistêmica que se observaram na avaliação hemodinâmica, mas o mecanismo mais provável é a diminuição da formação do cGMP pela inibição da guanilil ciclase.

Verificou-se que apenas as determinações de óxido nítrico mostraram o caráter inibitório do AM, mas os marcadores da SIRS e os parâmetros hemodinâmicos não tiveram comportamento uniforme. Deve-se ressaltar, no entanto, que a dose de AM utilizada foi muito baixa e isso pode ter influenciado nos resultados.

\section{CONCLUSÕES}

Nesse estudo prospectivo, randomizado, em pacientes submetidos à cirurgia de revascularização miocárdica com CEC, conclui-se que o uso do azul de metileno:

1. Não mostrou efeitos clínicos adversos especialmente nas trocas gasosas e hemodinâmica pulmonares.

2. Mostrou tendência a menor resposta inflamatória sistêmica pela avaliação dos níveis de TNF $\alpha$, número de leucócitos e neutrófilos. Os níveis das demais citocinas dosadas não mostraram diferenças significantes entre os pacientes que receberam ou não a infusão venosa do azul de metileno.

3. Mostrou importante ação de inibição da produção de óxido nítrico.

4. Mostrou valores significativamente maiores de resistência vascular sistêmica e pressão arterial sistêmica diastólica em um dos períodos de pós-operatório, sugerindo melhor estabilidade hemodinâmica.

\section{AGRADECIMENTOS}

Eliana Almeida G. Reis, Rita de Cássia Ribeiro Silva, Fernando de Queiroz Cunha e Giuliana Bertozi Francisco, pelas dosagens, computação e estatística do trabalho.

\section{REFERÊNCIAS BIBLIOGRÁFICAS}

1. Parker DJ, Cantrell JW, Karp RB, Stroud RM, Digerness SB. Changes in serum complement and immunoglobulins following cardiopulmonary bypass. Surgery 1972; 71:824-7.

2. Gomes WJ, Carvalho AC, Palma JH, Gonçalves Jr. I, Buffolo E. Vasoplegic syndrome: a new dilemma. J Thorac Cardiovasc Surg 1994; 107:942-3
3. Andrade JCS, Batista Filho ML, Évora PRB, Tavares JR, Buffolo E, Ribeiro EE et al. Utilização do azul de metileno no tratamento da síndrome vasoplégica após cirurgia cardíaca. Rev Bras Cir Cardiovasc 1996; 11:107-14.

4. Kofidis T, Strüber M, Wilhelmi M, Anssar M, Simon A, Harringer $\mathrm{W}$ et. al. Reserval of severe vasoplegia with singledose methylene blue after heart transplantation. J Thorac Cardiovasc Surg 2001; 122:823-4.

5. Koelzow H, Gedney JA, Baumann J, Snook NJ, Bellamy MC. The effect of methylene blue on the hemodynamic changes during ischemia reperfusion injury in orthotopic liver transplantation. Anesth Analg 2002; 94:824-9.

6. Grayling M, Deakin CD. Methylene blue during cardiopulmonary bypass to treat refractory hypotension in septic endocarditis. J Thorac Cardiovasc Surg 2003; 125:426-7.

7. Salaris SC, Babbs CF, Voorhees 3rd WD. Methylene blue as an inhibitor of superoxide generation by xanthine oxidase: a potential new drug for the attenuation of ischemia / reperfusion injury. Biochem Pharmacol 1991; 42:499-506.

8. Finkel MS, Oddis CV, Jacob TD, Watkins SC, Hattler BG, Simmons RL. Negative inotropic effects of cytokines on the heart mediated by nitric oxide. Science 1992; 257:387-9.

9. Mayer B, Brunner F, Schmidt K. Inhibition of nitric oxide synthesis by methylene blue. Biochem Pharmacol 1993; 45:367-74.

10. Brasil LA, Gomes WJ, Salomão R, Buffolo E. Inflammatory response after myocardial revascularization with or without cardiopulmonary bypass. Ann Thorac Surg 1998; 66:56-9.

11. Chenoweth DE, Cooper SW, Hugli TE, Stewart RW, Blackstone EH, Kirklin JW. Complement activation during cardiopulmonary bypass: evidence for generation of C3a and C5 a anaphylatoxins. N Engl J Med 1981; 304:497-503.

12. Cremer J, Martin M, Redl H, Bahrami S, Abraham C, Graeter $\mathrm{T}$ et al. Systemic inflammatory response syndrome after cardiac operations. Ann Thorac Surg 1996; 61:1714-20.

13. Diegeler A, Doll N, Rauch T, Haberer D, Walther T, Falk V et al. Humoral immune response during coronary artery bypass grafting: a comparison of limited approach, "off-pump" technique, and conventional cardiopulmonary bypass. Circulation 2000; 102 [19 suppl 3]: III 95-100.

14. Edmunds Jr. LH. Inflammatory response to cardiopulmonary bypass. Ann Thorac Surg 1998; 66 (5 suppl) S12-6.

15. Holmes JH, Connolly NC, Paull DL, Hill ME, Guyton SW, Ziegler SF et al. Magnitude of the inflammatory response to cardiopulmonary bypass and its relation to adverse clinical outcomes. Inflamm Res 2002; 51:579-86. 
16. Taylor KM. SIRS: the systemic inflammatory response syndrome after cardiac operations. Ann Thorac Surg 1996; 61:1607-8

17. Westaby S. Complement and the damaging effects of cardiopulmonary bypass. Thorax 1983; 38:321-5.

18. Bone RC, Balk RA, Cerra FB, Dellinger RP, Fein AM, Knaus WA et al. Definitions for sepsis and organ failure and guidelines for the use of innovative therapies in sepsis. Chest 1992; 101:1644-55

19. Évora PR. Should methylene blue be the drug of choice to treat vasoplegias caused by cardiopulmonary bypass and anaphylactic shock? J Thorac Cardiovasc Surg 2002; 119:632-4.

20. Keaney Jr. JF, Puyana JC, Francis S, Loscalzo JF, Stamler JS, Loscalzo J. Methylene blue reverses endotoxin-induced hypotension. Circ Res 1994; 74:1121-5.

21. Preiser JC, Lejeune P, Roman A, Carlier E, De Backer D, Leeman $\mathrm{M}$ et al. Methylene blue administration in septic shock: a clinical trial. Crit Care Med 1995; 23:259-64.
22. Schneider F, Lutun P, Hasselmann M, Stoclet JC, Tempé JD. Methylene blue increases systemic vascular resistance in human septic shock: preliminary observations. Intensive Care Med 1992; 18:309-11.

23. Weingartner R, Oliveira E, Oliveira ES, Sant'Anna UL, Oliveira RP, Azambuja LA et al. Blockade of the action of nitric oxide in human septic shock increases systemic vascular resistance and has detrimental effects on pulmonary function after a shost infusion of methylene blue. Braz J Med Biol Res 1999; 32:1505-13.

24. Brett SJ, Quinlan GJ, Mitchell J, Pepper JR, Evans TW. Production of nitric oxide during surgery involving cardiopulmonary bypass. Crit Care Med 1998; 26:272-8.

25. Aldea GS, Soltow LO, Chandler WL, Triggs CM, Vocelka CR, Crockett GI et al. Limitation of thrombin generation, platelet activation, and inflammation by elimination of cardiotomy suction in patients undergoing coronary artery bypass grafting treated with heparin-bonded circuits. J Thorac Cardiovasc Surg 2002; 123:742-55. 